\title{
Computations in Plasma Physics
}

\section{Biskamp, Garching}

(Max-Planck-Institut für Plasmaphysik)

Plasma physics plays an important role in astrophysics as well as in nuclear fusion research. It is a complex field where a great variety of different physical effects occur and interact.

It is convenient to distinguish several levels of description of plasma behaviour: a) MHD theory. To describe the global properties of a plasma configuration, e.g. the discharge column in a fusion experiment, a fluid model is most suitable, the magnetohydrodynamic (MHD) theory. It accounts, in particular, for the intimate connection between the plasma and magnetic field resulting from the high electrical conductivity;

b) Microscopic theory. A hot, dilute plasma may be in a state which strongly differs from local thermodynamic equilibrium, and hence is susceptible to velocity space instabilities or micro-instabilities. This implies excitation of certain collective modes to some level of microscopic turbulence which may give rise to transport effects far exceeding those due to particle collisions. These processes are described by the Vlasov, or collisionless Botzmann equation;

c) Transport theory. In nuclear fusion research the understanding of transport across the confining magnetic field is very important. Many different processes occur and influence each other; plasma diffusion, electron and ion thermal conduction, particle interactions, penetration and radiation of impurity ions. These and other effects are described by a complex system of coupled transport equations.

As at each level of description one has to deal with multi-dimensional differential or integro-differential equations, which in general are strongly non-linear, and have complicated boundary conditions in complex geometries, analytical methods such as perturbation theory can provide only a qualitative answer at best.

With the advance of powerful scientific computers in the past decade, numerical computing has entered practically all fields

Fig. 1 - Stellarator plasma equilibrium configuration.

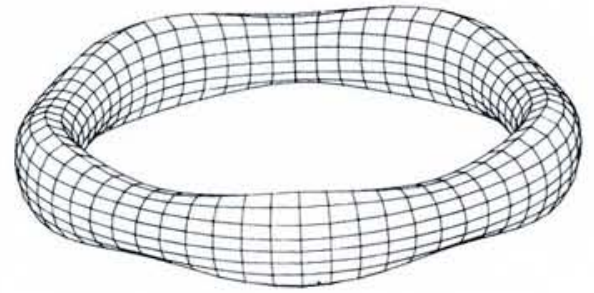

of plasma physics. On the one hand, it has become a powerful instrument in the evaluation as well as the planning of experiments; on the other, our theoretical understanding of basic plasma phenomena is now achieved to a large extent through computer simulations.

\section{MHD Computations}

In MHD theory, the plasma is considered to be an electrically conducting fluid of mass density $\rho$, pressure $p$ and velocity $\vec{v}$. It couples to the magnetic field $\vec{B}$, which is, at least in part, generated by electrical currents within the plasma itself. The MHD equations are:

$$
\partial \rho / \partial t+\nabla \cdot \rho \vec{v}=0,
$$

$\partial \rho \vec{v} / \partial t+\nabla \cdot \rho \vec{v} \vec{v}=-\nabla p+(\nabla \times \vec{B}) \times \vec{B}$,

$$
\partial p / \partial t+\nabla \cdot p \vec{v}=-(\gamma-1) p \nabla \cdot \vec{v},
$$
$\partial \vec{B} / \partial t-\nabla \times(\vec{v} \times \vec{B})=\nabla \times \eta(\nabla \times \vec{B})$.

Equation (4) is Faraday's law, where the generalized Ohm's law $\vec{E}=-\vec{v} \times \vec{B}+$ $\eta \vee \times \vec{B}$ has been used to eliminate the electric field. In many cases, the plasma resistivity $\eta$ may be neglected. Equations (1) - (4) are then called ideal MHD theory. Equilibrium and stability are usually investigated in this framework. If dynamic processes take place, a small but finite resistivity may, however, play an important role.

\section{MHD Equilibrium}

A basic problem is the calculation of equilibrium configurations. In nuclear fusion research, for instance, it is necessary to find configurations with a high plasma pressure for a given externally applied magnetic field. In equilibrium, magnetic and pressure forces balance each other:

$$
\vec{F} \equiv-\nabla p+(\nabla \times \vec{B}) \times \vec{B}=0
$$

The solution of this simple-looking equation, together with the condition $\nabla \cdot \vec{B}=0$, in general constitutes a very difficult problem. Fortunately, in many cases of interest, the symmetry of the configuration simplifies the problem to a twodimensional one, reducing eq. (5) to a single equation for $\psi$, the component of the vector potential in the direction of the ignorable coordinate, which is of the form

$$
L \psi=G(\psi) \text {. }
$$

Here $L$ is a linear differential operator (a generalization of the two-dimensional Laplacian), and $G$ a functional of $\psi$. Surfaces of constant $\psi$ contain the magnetic field lines and are hence called magnetic surfaces. $G$ contains two free functions, one being the pressure assignment to each magnetic surface. In most cases of interest, eq. (6) can only be treated numerically; efficient codes have been developed, in particular for axial and helical symmetry, both of which play an important role in fusion research. As $G$ is, in general, nonlinear in $\psi$, the solution of (6) requires the use of iteration methods. Special techniques have to be applied to cope with the possibility of multiple solutions.

Whereas two-dimensional equilibria are now calculated routinely, the calculation of fully three-dimensional equilibria, such as those appearing in stellarator magnetic plasma confinement, is much more involved, and the first numerical attempts date back only a few years. Apparently a solution of eq. (5) can be obtained only by a relaxation procedure, where starting from an initial guess at the configuration, the net force $\vec{F}$ is gradually reduced to zero. The procedure becomes clear from the equivalent energy formulation. The initial distribution of $p$ and $\vec{B}$ is adjusted under the constraints implied by the MHD equations in such a way that the plasma energy

$$
W=\int d^{3} \times\left[B^{2} / 2+p /(\gamma-1)\right]
$$

decreases monotonically. $A$ state of minimum energy is thus approached which represents an MHD equilibrium, the Euler equation of the variational principle $\delta W=$ 0 being just eq. (5). Several codes have been developed. They differ primarily in the choice of coordinates, using either a fixed Eulerian grid or a semi-Lagrangian scheme by treating the instantaneous flux surfaces as coordinate surfaces. The latter approach is inherently more accurate, but leads to special numerical problems and is restricted to configurations with only one magnetic axis. Various relaxation schemes have been developed in order to minimize the number of iterations. It is clear from the fact that eight functions defined on a threedimensional grid have to be followed over many iterations, that a numerical solution of this problem is only just marginally possible, even on the largest existing computers. For instance, mesh grids are limited to about $30^{3}$ points. Fig. 1 gives an example of a computed stellarator equilibrium.

\section{MHD Stability}

An equilibrium configuration is, however, physically relevant only if it is at least locally stable. It is true that, by minimizing the energy (7), the three-dimensional codes lead in principle to a stable equilibrium. In practice, however, the iteration procedure does not allow for certain modes or displacements. These have to be excited explicitly to check for stability. Generally speaking, 
the three-dimensional computations are still too coarse to allow reliable statements to be made about the stability of the resulting equilibria.

The two-dimensional equilibrium codes solving equations of type (6) do not distinguish between stable and unstable configurations. Their stability therefore has to be investigated separately. In recent years, numerical stability codes have been developed which allow computation of the full spectrum of perturbation eigenmodes for almost any numerically generated equilibrium. Discretizing the corresponding linearized MHD equations on a suitably chosen grid, and making the ansatz $\exp (-i \omega t)$ for the perturbation, the problem takes the form of the matrix eigenvalue equation

$$
\underline{A} \underline{\underline{x}}-\omega^{2} \underline{\underline{B x}} \underline{\underline{x}}=0 .
$$

Here $\underline{\underline{A}}, \underline{\underline{B}}$ are Hermitian matrices, hence the eigenvalues $\omega^{2}$ are real. For $\omega^{2}>0$ the frequency $\omega$ is real, the mode oscillates with constant amplitude, while for $\omega^{2}<0$, there is a mode which can grow exponentially. Hence the equilibrium is unstable if and only if there are negative eigenvalues $\omega^{2}$. As $\underline{\underline{A}}, \underline{\underline{B}}$ are sparse matrices of huge dimensions, considerable effort must be made to reorder them so as to reduce the problem to a tractable form. Rather precise eigenvalues are obtained by solving the system (8) with different grids and extrapolating these results to zero grid size. Fig. 2 shows an unstable axisymmetric (tokamak) equilibrium and the structure of the most unstable mode. The arrows depict the (normalized) unstable plasma motion.

Fig. 2 - a) Axisymmetric (tokamak) equilibrium with coordinate lines suitably chosen for stability computation (radial coordinate lines are flux surfaces $\psi=$ const);

b) Structure of the most unstable eigenmode.

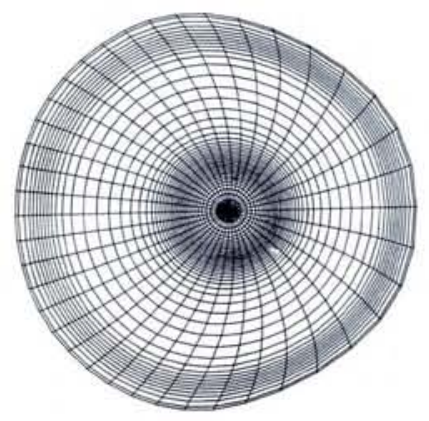

a)

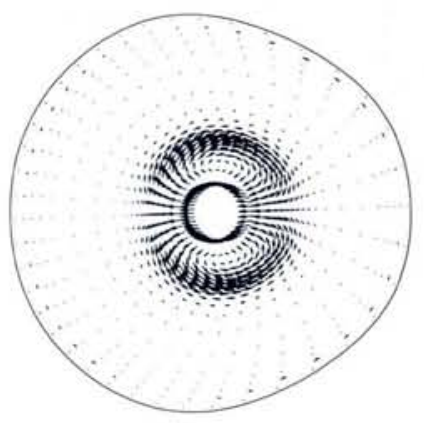

NI $\widehat{\text { KHEF }}$

\section{EXPERIMENTAL PHYSICIST (PhD)}

to participate in electron scattering experiments.

The Institute has recently started operation of its $500 \mathrm{MeV}$ high duty cycle electron linac (MEA).

Experimental equipment comprises two magnetic spectrometers that offer both resolution (10-4) single-arm and a two-arm coincidence mode of operation.

Applicants are expected to have experience in experimentation with complex particle-detection apparatus.

Experience in the field of nuclear physics is required.

The appointment is for a maximum of 2 years.

\section{Additional information may be obtained from \\ Prof. dr. C. de Vries, \\ Post-Box 4395 \\ 1009 AJ AMSTERDAM, The Netherlands \\ (phone Amsterdam 5922142).}

Please address your applications, including a career resume and the names of three referees within 4 weeks after appearance of this advertisement to

\author{
NIKHEF-, \\ Personnel office, \\ Postbus 41882, \\ 1009 DB AMSTERDAM.
}

\section{Dynamical MHD Processes}

In physical reality, unstable equilibria cannot persist and hence their investigation appears to be of purely academic interest. However, it often occurs that an instability threshold is exceeded during the slow quasistatic change of a configuration. The structure of the most unstable mode indicates then the manner in which the system will initially evolve from the original configuration. In many cases, the state into which the system eventually settles is a neighbouring equilibrium of somewhat lower energy: the instability threshold is just a bifurcation point. But there are also situations where passage into an unstable regime gives rise to catastrophic behaviour which completely destroys the original configuration, leading to a strongly turbulent state. Explosive phenomena such as disruptions in tokamak plasmas or solar flares are associated with such behaviour.

To describe these phenomena, numerical simulations of the time-dependent MHD equations ( 1 ) $-(4)$, have been rather successful. Finite resistivity has to be considered, even if formally very small, as it allows for changes in magnetic field topology. The MHD equations are conveniently treated in a simplified form that makes use of the small value of the plasma pressure in many applications; this not only reduces the number of unknown functions, but also strongly relaxes the restriction on the time step by eliminating the fastest waves from the simulated system. Such numerical simulations can explain the characteristic features of tokamak disruptions. They reveal, for instance, the origin of rapid electron energy loss, namely the large-scale destruction of magnetic surfaces by ergodization of field lines (see Fig. 3 overleaf).

\section{Microscopic Theory}

In a hot, dilute plasma, which is of particular interest in both fusion research and astrophysics, the effects of individual particle collisions are weak, and thus substantial deviations of the velocity distribution from the Maxwellian form occur. Small groups of particles in velocity space can interact resonantly with the natural modes of the plasma. They may either transfer energy to a wave and thus lead to growth of the wave amplitude, this process being called a micro-instability, or extract energy from the wave, leading to anomalous wave absorption. The non-linear behaviour, e.g. the level and spectrum of the turbulence thus excited, and the energy distribution of the resonantly heated particles, is determined by the particle motions in phase space. The equation describing a collisionless plasma is the Vlasov equation for the distribution 

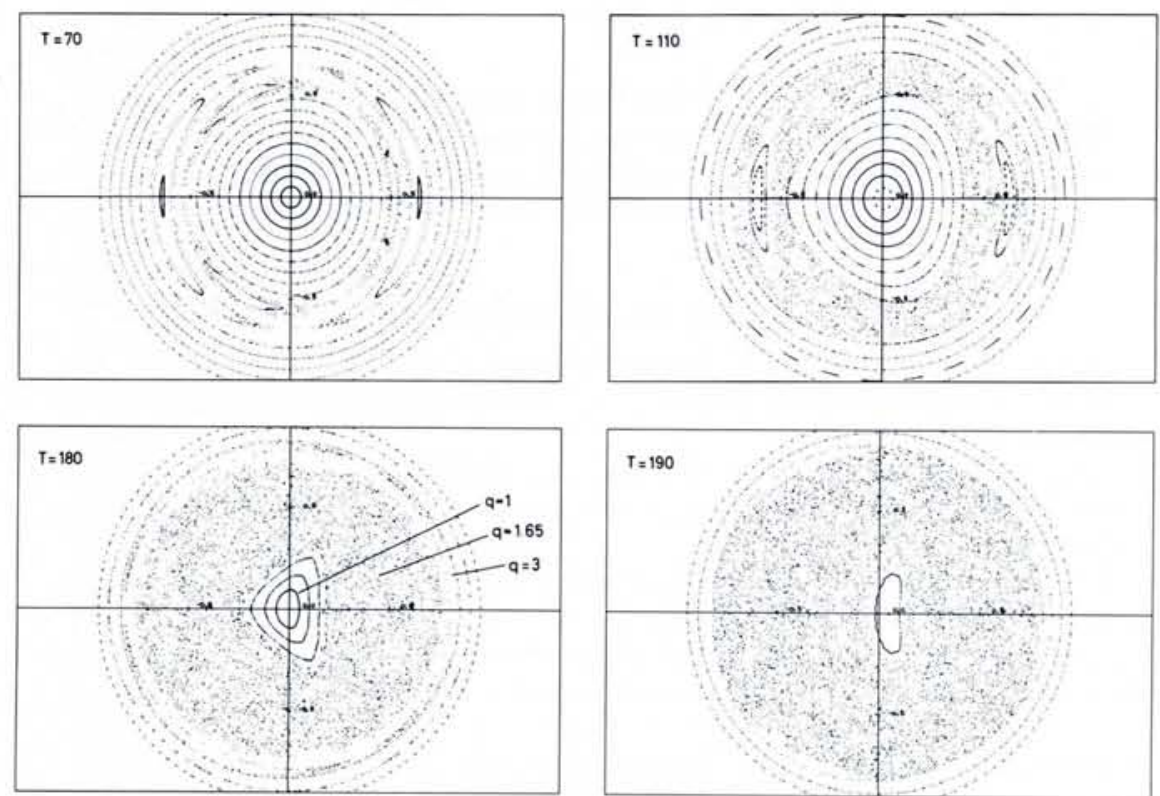

Fig. 3 - Time sequence of the behaviour of the plasma cross-section in a computed simulation of a tokamak disruption, illustrating the destruction of magnetic surfaces. T is measured in Alfven times.

function $f_{\alpha}(\vec{x}, \vec{v}, t)$ for each particle species $\alpha$

$\partial f_{\alpha} / \partial t+\vec{v} \cdot \partial f_{\alpha} / \partial \vec{x}+\left(e_{\alpha} / m_{\alpha}\right)(\vec{E}+\vec{v} \times \vec{B})$

. $\partial f_{\alpha} / \partial \vec{v}=0$

where the self-consistent fields $\vec{E}, \vec{B}$ follow Maxwell's equations

$\nabla \cdot \vec{E}=\rho, \quad \nabla \times \vec{B}-\partial \vec{E} / \partial t=\vec{J}$,

$\nabla \times \vec{E}+\partial \vec{B} / \partial t=0$

with the charge and current densities

$$
\rho=\sum_{\alpha} e_{\alpha} \int d^{3} v f_{\alpha}, \vec{J}=\sum_{\alpha} e_{\alpha} \int d^{3} v \vec{v} f_{\alpha} .
$$

Only in the linearized form may these equations be investigated by analytical methods. The non-linear equations require a numerical treatment, the most widely used approach being particle simulation. In contrast to molecular dynamics computations in the theory of liquids, individual particle interactions are negligible here compared with collective effects. One may therefore compute the fields on a fixed Eulerian grid of rather coarse mesh size. Particles move across this grid under the influence of the local fields, which in turn are determined by the distribution of the particles. Charge and current densities are obtained by counting particles and adding their velocities in each grid cell.

To reduce numerical noise, fields are interpolated between grid point values and particles are given a finite size. This method of particle simulation is called the "particlein-cell" (PIC) method. In spite of the formally rather poor accuracy of this MonteCarlo type of approach, in practice, the method is often found to have a good signal-to-noise ratio even for small numbers of particles per cell, and it is the only feasible method for multi-dimensional problems.

Because of the large variety of modes and micro-instabilities with widely different space and time scales, several versions of the PIC method are in use.. We primarily distinguish between high and low-frequency processes.

\section{High-frequency Phenomena}

High-frequency oscillations in a plasma are predominantly electrostatic with frequencies either close to the plasma of Langmuir frequency $\omega_{\mathrm{pe}}$ or the ion sound frequency $k c_{\mathrm{s}} \leq \omega_{\mathrm{pi}}$, where $c_{\mathrm{s}}$ is the acoustic speed and $\omega_{\mathrm{pi}}$ the ion plasma frequency. Wavelengths are of the order of the Debye length or some equivalent spatial scale length. Electron beams penetrating into a plasma are the main cause of excitation of Langmuir wave turbulence. The ions do not participate in the oscillatory motion and hence can be considered immobile, most conveniently as a smooth background of positive charge density. If the relative flow of electrons and ions, which gives rise to an electric current, exceeds a threshold, ion sound waves are excited. Ion sound turbulence leads to an anomalous electrical resistivity, an important phenomenon in weakly collisional plasmas. As both electrons and ions interact resonantly with the waves, both particle species have to be considered. The large ion-electron mass ratio poses a major computational problem. To reduce the number of time steps required in a typical run, the mass ratio is artificially reduced in most simulation calculations. By varying $m_{i} / m_{e}$ one tries to discover the $m_{i} / m_{\mathrm{e}}$ scaling law, which in principle then allows extrapolation to realistic values $>10^{3}$.

Langmuir and ion sound waves are also excited by intense electromagnetic waves, as occur during the interaction of a plasma with laser radiation. As they lead to anomalous backscattering of laser energy, these processes play an important role in laser fusion research. Large-scale two-dimensional simulations have been performed with up to $10^{6}$ particles followed over $10^{5}$ time steps and taking tens of hours on the most powerful computers. Fig. 4 illustrates typical diagnostics of a particle simulation run. It displays the modulation of the spatial electron distribution due to plasma waves parametrically excited by an intense light wave.

\section{Low-frequency Phenomena}

If wave frequencies are much smaller than natural frequencies such as the Langmuir frequency or the cyclotron frequency, i.e. the frequency of particle gyration in the magnetic field, approximations to the exact PIC description of the plasma are often very useful, e.g. the representation of the electrons by a fluid without inertia, or the guiding centre approximation for particle orbits which results from averaging the true orbits over a cyclotron period. A particularly important class of low-frequency waves in magnetic confinement devices are so-called drift modes, which are due to the (unavoidable) presence of density and pressure gradients. At high plasma temperatures, these modes can be driven unstable by wave particle resonances and hence require a microscopic description. Because of the geometrical effects involved, the problem is inherently three-dimensional in space, which makes particle simulations very expensive and just barely feasible to date.

Another area of low-frequency phenomena comprises magnetic collisionless shock waves such as the bow shock in front of the earth's magnetosphere. Particle simulations can account for the reflec-

Fig. 4 - Excitation of plasma waves due to Raman backscattering and sidescattering of laser light incident on a a) linear, b) exponential plasma density profile. $n_{c}$ critical density.

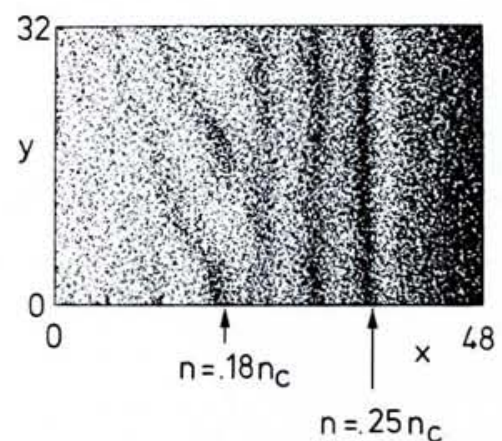

a)

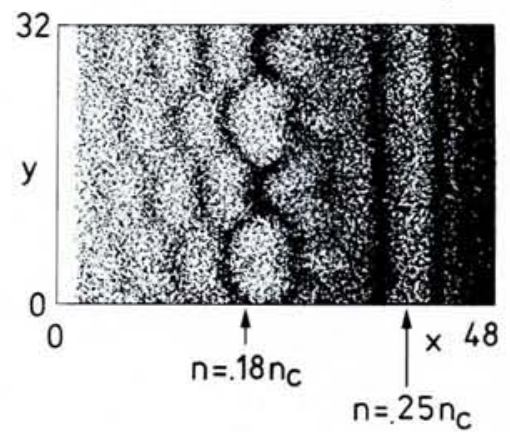


ted ions observed in the upstream solar wind plasma, the slow thermalization of the ions on the downstream side, as well as the strong magnetic field turbulence.

\section{Transport in Toroidal Magnetic De- vices}

In fusion research, toroidal magnetic traps (tokamak, stellarator, etc.) play a dominant role. In these devices, closed magnetic surfaces provide unlimited single particle confinement. Because of the presence of collisions and electric and magnetic fluctuations, however, a slow diffusion of particles and energy across magnetic surfaces takes place. To minimize these losses is one of the main objectives of fusion research.

Computer models of cross-field transport are using an idealized geometry by allowing for only one spatial coordinate, the minor radius of the toroidal configuration. Deviation from this symmetry due to a small toroidal aspect ratio, non-circular plasma cross-section, or toroidal field ripple, are neglected or accounted for phenomenologically. On the other hand, the maximum possible number of transport processes is considered, plasma diffusion, ion and electron thermal conduction, neutra particle fluxes, transport of impurity ions, radiation losses and others. All of these processes have to be treated simultaneously in a transport code which is, as a result, highly complex. The quality of such a code is judged in a twofold way:

a) from a numerical point of view, i.e. by the reliability and efficiency of the numerical models in describing the corresponding physical effects; b) by comparison with experimental observations.

Regarding the latter, it has been known for a long time that some transport processes in a tokamak, in particular electron heat conduction, cannot be accounted for by collisional effects and to obtain agreement with the experiments, phenomenological transport coefficients were introduced. Comparison with measurements of local quantities such as density and temperature profiles, gives rather precise information on the magnitudes and scaling laws of the anomalous transport processes, which facilitates the search for the physical origin of these anomalies. For example, the strongly enhanced electron thermal transport now appears to be due to magnetic microfluctuations, which lead to a certain roughness of the magnetic surfaces.

From the numerical view point, the computation of neutral particle behaviour is very involved. As these particles are not affected by the magnetic field, the description of their penetration into the plasma, ionization and charge exchange is inherently two-dimensional. To date, the most reliable numerical approach is provided by Monte Carlo calculations. Since the radial transport of neutrals is much more rapid than that of charged particles, each time step on the latter scale requires a complete calculation of the distribution of neutrals. Not surprisingly, this part of the program is by far the most time-consuming.

In a fusion plasma, all ions other than the fusionable isotopes of hydrogen are called impurities, as they have a detremental effect. In particular, heavy impurities which are not completely ionized cause severe losses due to line or recombination radiation. Knowledge of the penetration of impurities into the plasma and their distribution in the plasma is thus of crucial importance. To describe the behaviour of ions such as iron sputtered off the confining walls, a large variety of data from atomic physics is required, e.g. ionization crosssections of highly ionized states, which are either only poorly or not at all known. It is evident that a full treatment of just the most dangerous impurities is extremely tedious, if only because of the enormous number of ionization states that have to be considered as different particle species. At present only light impurities such as oxygen are described satisfactorily, while heavy ions are still handled in a very approximate way. Transport calculations play an indispensable part in the planning of new fusion experiments, although presentday knowledge of many transport effects is still so poor that such predictions of plasma behaviour can only be regarded as a first guess.

This short review of numerical computations in plasma physics could only mention the major fields where large-scale computations are being performed. Though the classical method of analytically deriving an approximate "formula" for a particular phenomenum will certainly remain useful, for many problems in plasma physics only the extensive use of numerical calculations or numerical simulations has provided reliable quantitative results and narrowed the gap between experimental observations and theoretical interpretation and prediction.

\section{New Individual Members}

Individual Members of the European Physical Society approved by Council in the Summer.

\section{CATEGORY 4A}

H. Bodin, Abingdon, UK

1. Bozovic, Belgrade, YU

M.A. de Pretis Cagnodo, Frascati, I

J.V. Feitzinger, Bochum, D

\section{CATEGORY 4C}

Austrian Physical Society

R. Schrittwieser, Innsbruck

F. Widder, Graz

Danish Physical Society

I. Balslev, Odense

H. Bøggild, Copenhagen

V. Frank, Lyngby

J. Hertz, Copenhagen

J.K. Kjems, Roskilde

P.L. Ølgaard, Lyngby

P. Thomsen, Lyngby

Eötvős Lorand Physical Society

F. Beleznay, Budapest

J. Harsanyi, Budapest

K. Lelkes, Budapest

F. Mezei, Brignoud, $F$

I. Pozsgai, Budapest

G. Remenyi, Budapest
German Physical Society

B. Brandt, Nieuwegein, NL S. Islam, Göteborg, S K.P. Lieb, Göttingen

Ch. Methfessel, Witten

F. Münnich, Braunschweig

H. Pallasch, Hitzacker

W. Richter, Ulm

R. Wienecke, Stuttgart

P. Zimmermann, Berlin

Hellenic Physical Society

G. Kapoulitsas, Thessaloniki

The Institute of Physics

J.M. Cassels, Liverpool

W. McGinty, Portsmouth

D.H. Parkinson, Malvern

P.A. Rodgers, Milton Keynes

D.A. Sweetman, Abingdon

Italian Physical Society

G. Benedek, Milan

A. Giardini-Guidoni, Frascati

M. Guzzi, Milan

M. Inguscio, Pisa

P. Veltri, Roges

The Netherlands' Physical Society

J. Los, Weesp

L. Niesen, Eelde

I. Silvera, Amsterdam

J. Reuss, Nijmegen

D. Schram, Eindhoven

J.T.M. Walraven, Amsterdam
Norwegian Physical Society

A. Eide, Kristiansand

O. Engvold, Oslo

A. Henriquez, Osio

A. Kildal, Oslo

S. Løvold, Kjelle

K. Olaussen, Trondheim

J. Samseth, Trondheim

Physical Section, Union of Czechoslovak Mathematicians and Physicists

R. Durny, Bratislava

L. Smrcka, Prague

Physical Section, Union of Yugoslav Societies of Mathematicians, Physicists and Astronomers

D. Sijacki, Belgrade

Polish Physical Society

A. Kiejna, Wrockaw

A. Warczak, Cracow

B. Warczak, Cracow

Swedish Physical Society

A. Jarmen, Västra Frölunda

D. Lundqvist, Bromma

A. Östlund, Ursviken

N. Svantesson, Göteborg

Turkish Physical Society

E. Inönü, Istanbul 